\title{
The Role of Delay and Connectivity in Throughput Reduction of Cooperative Decentralized Wireless Networks
}

\author{
Ahmed Alkhayyat, ${ }^{1}$ Orhan Gazi, ${ }^{1}$ and Sattar B. Sadkhan ${ }^{2}$ \\ ${ }^{1}$ Department of Electronics and Communication Engineering, Cankaya University, 06530 Ankara, Turkey \\ ${ }^{2}$ Department of Information Technology, University of Babylon, Babylon, Iraq \\ Correspondence should be addressed to Ahmed Alkhayyat; al_kuptan@yahoo.com
}

Received 18 January 2015; Revised 28 March 2015; Accepted 28 March 2015

Academic Editor: Bo Shen

Copyright (C) 2015 Ahmed Alkhayyat et al. This is an open access article distributed under the Creative Commons Attribution License, which permits unrestricted use, distribution, and reproduction in any medium, provided the original work is properly cited.

We proposed a multiple relay selection protocol for decentralized wireless networks. The proposed relays selection protocol aims to address three issues: (1) selecting relays within the coverage area of the source and destination to ensure that the relays are positioned one hop away from the destination, (2) ensuring that the best node (best relays with less distance and attenuation from the destination) access the channel first, and (3) ensuring that the proposed relays selection is collision-free. Our analysis also considers three important characteristics of decentralized wireless networks that are directly affected by cooperation: delay, connectivity, and throughput. The main goal of this paper is to demonstrate that improving connectivity and increasing number of relays reduce the throughput of cooperative decentralized wireless networks; consequently, a trade-off equation has been derived.

\section{Introduction}

Despite the advantages of multiple antennas, it is not practical to use multiple antennas for a mobile set becaue of the size and costs of handsets of decentralized wireless networks (ad hoc) or cellular networks. A solution to this problem is the cooperative communication systems (CC), in which the source sets multiple antennas virtually; for example, the source broadcasts the data to the neighbor nodes (relays) and to the destination, and then the relays retransmit the received data to the destination, and the destination combines all received data from the source and relays.

Two categories of cooperation have been considered in the literature: multihop cooperation protocol (MCP) [1] and load distributed cooperation protocol (LDCP) [2-4]. In MCP, the source identifies the nodes near to itself and near to the destination. These nodes are also called relays. When the source locates such relays, it transmits data to the relays, and the relays retransmit the data to the destination. The purpose of relays in MCP is to avoid the signal attenuation associated with direct transmission, that is, source destination transmission. A code distribution protocol is an approach of LDCP, by which the source divides the redundant bits into two parts instead of transmitting the full redundant bits to the destination; the first part is transmitted by the source to the destination and relays, and the second part is transmitted by the relays to the destination. The key difference between MCP and LDCP is that in MCP the destination receives data transmitted by the source merely, while in LDCP the destination receives data from the source and relays. In fact, the LDCP is associated with a longer delay than MCP, consequently, decreased throughput; however, LDCP is associated with high diversity gain compared to MCP. In this paper, we have considered code distribution protocol as an example of LDCP.

The advantages of LDCP include the improved detection capability at the receiver, connectivity, and reduced bit error rate, while its disadvantages include delay, synchronization difficulties, and reduced rate. LDCP enhances connectivity by improving the received signal-to-noise ratio through the combination of the received data from the source and multiple relays via maximal ratio combining (MRC). Such a combination increases the size of the coverage area of the source and it also increases the prohibited area size (more nodes share single time slots); on the other hand, the delay increased because the data were forwarded to relays, 
and relays forward the received data to the destination. Therefore, the throughput is sacrificed because of the delay and connectivity enhancement.

CC in decentralized wireless networks (DWN) or infrastructure less networks requires redesign in their protocols or planning new protocols to use CC efficiently. One of the efficient protocols that can improve the LDCP is the relay selection because if LDCP was employed and node was selected randomly, the communication system would become worse compared to system that does not use LDCP. Hence, an optimal relays selection is required. Optimal relays selection has been studied recently in [5-7], and delays in CC have been studied in $[8,9]$. In the DWN, the LDCP has a negative effect on throughput because of delays and connectivity enhancement. Meanwhile, delay and throughput trade-off have been studied in [10], and the trade-off between throughput and connectivity has been studied in [11]. Most of the previous works on LDCP which are based on the backoff time function to select best relay did not address several issues $[9,12]$ : (1) how to reduce the delay to enhance the throughput but neglect the connectivity effect; (2) protocols are based on single relay selection; (3) only relays within the source and destination coverage area are considered; (4) network coding can be used to enhance the throughput which burdens the destination to separate received frames.

To address the above missing issues such as the best relays selection, delay, connectivity, and throughput all together, we propose a cooperative medium access (CMAC) protocol based on slight changes in CSMA/CA with RTS/CTS packets. The contributions of this work are summarized as follows: (1) we propose a backoff time function (BTF) in which the best relays have a smaller backoff time and bad relays have a larger backoff time; (2) we propose a CMAC protocol that reduces delay, prevents extra negotiation via RTS/CTS packets, and is collision free; (3) our proposed protocol employs multiple relay selection; (4) we analysed the connectivity when LDCP is employed in terms of the linking probability and node degree; (5) we inspected the throughput when LDCP is employed. We also demonstrated that throughput decreases by increasing the delay and enhancing the connectivity; consequently a trade-off is provided. This point is the main goal of this paper.

The rest of the paper is outlined as follows: we explained in detail the proposed cooperative MAC protocol, including basics of the code distribution protocol, BTF, and relay contention collision-free in Section 2. In Section 3, proposed cooperative medium access delay is analysed. In Section 4, we analysed connectivity of the LDCP by considering linking probability and node degree. In Section 5, we analysed the effect of increased delays and enhanced connectivity on throughput and a trade-off conclusion is drawn. In Section 6 the performance results are discussed. Finally, Conclusions are drawn in Section 7 and future work is stated.

\section{Proposed Cooperative MAC Protocol}

In this paper, the function of a CMAC is to choose the best relays with good channel parameters to the destination

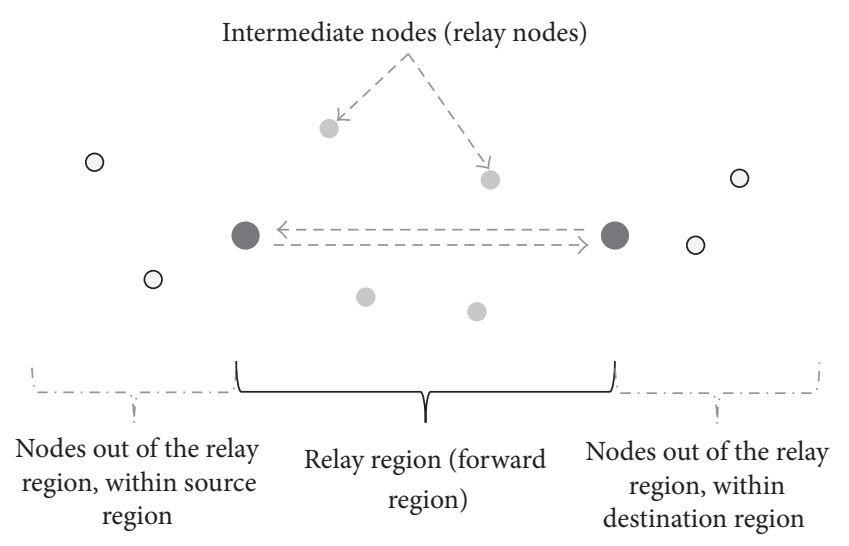

FIgURE 1: A scenario of communication and relays location.

only. However, source to relays channel wasn't considered in our analysis because the relays that decode the retrieved frames correctly can participate in cooperation. Otherwise they stay silent. In this paper, we propose a CMAC protocol that is based on slight changes to the IEEE802.11 distributed coordination function (DCF) with CSMA/CA with RTS/CTS packets $[13,15]$ and address several potential problems associated with cooperation as listed below.

(1) Relay node location or relay region (RR) selection, see Figure 1: it is important to select relays with direct transmission to a destination rather than relays that are two hops away from the destination. Simply, we select only relays that fall within the RR of the transmission ranges of both the source and destination. Selecting relays one hop from the destination can prevent extra delays caused by multiple hops (two or more); that is, if the time required to transmit data from over single-hop relay is $T$, then the time required to transmit the data over two-hop relay is $2 \mathrm{~T}$.

(2) Channel access schemes: in DWN, controlling access of relays is significant. Two issues are important to propose a good channel access scheme: (a) the relays must be selected rapidly to prevent delay; (b) the relays with the best channel quality to the destination must access the channel first before relays of inferior quality to ensure superior performance.

(3) Collision-free: LDCP inherently increases the delay in the DWN; thus, we must redesign the MAC protocol to prevent collisions between relays in order to reduce the delay.

2.1. Channel and Signal-to-Noise Ratio Formulation. In this paper, we consider distributed convolutional code protocol (DCCP) as an approach of code distribution protocol; the DCCP is similar to the protocol in [16] with a simple modification to be applicable for DWN. This modification prevents the source from transmitting in MAC mode to the destination in order to reduce the delay in CC. In what follows, we indicate the source, $k$ th relay, and destination nodes by $S, R_{k}$, and $D$, respectively, as well as indicating 


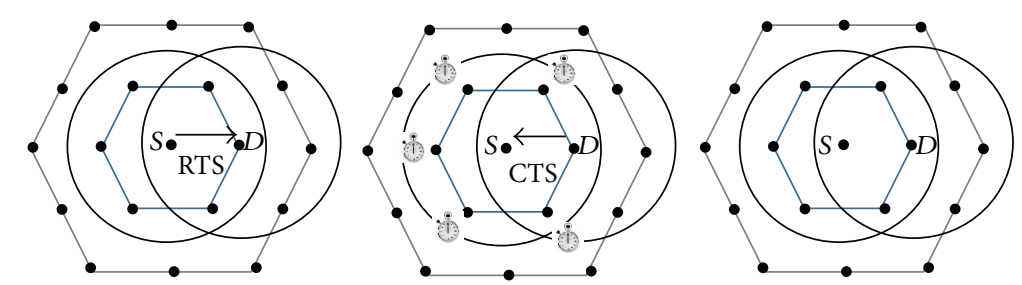

FIGURE 2: RR selection steps based on the RTS/CTS packets of the proposed protocol.

the direct transmission mode (i.e., noncooperative mode) and cooperative communication mode by DTM and CTM, respectively. DCCP works in two modes: broadcasting (BC) mode followed by medium access control (MAC) mode. In the BC mode, $S$ broadcasts the first frame (FF) with half redundant bits $N_{b}$ to $R_{k}$ and $D$. Therefore, the received signals at $R_{k}$ and at $D$ are given as

$$
\begin{gathered}
y_{S R_{k}}=\sqrt{R_{b} P_{b}}\left(d_{S R_{k}}\right)^{-\lambda} x+\eta_{S R_{k}}, \\
y_{S D}=\sqrt{R_{b} P_{b}}\left(d_{S D}\right)^{-\lambda} x+\eta_{S D},
\end{gathered}
$$

where $R_{b}$ and $P_{b}$ are the coding rate and power transmitted in the BC mode, respectively; $d_{S D}$ and $d_{S R_{k}}$ are the distances from $S$ and $R_{k}$ to destination, respectively; $\lambda$ is the path loss exponent that varies from 2 to 6 [17]; $x$ and $\eta_{S R_{k}}$ with $\eta_{S D}$ are the modulated signal and the complex white noise with zero mean and unit variance from $S$ to $R_{k}$ and from $S$ to $D$, respectively. In the MAC mode, if $R_{k}$ decodes the received FF correctly (the FF that was transmitted by $S$ in BC mode), then it reencodes the FF with half redundant bits named $N_{\mathrm{mc}}$ and it retransmits the encoded SF to the $D$ (i.e., $N_{\text {DTM }}=N_{b}+N_{\text {mc }}$, $N_{\text {DTM }}$ is the total redundant bits of DTM), where in the MAC mode the received signals at $D$ are given as

$$
y_{R_{k} D}=\sqrt{R_{\mathrm{mc}} P_{\mathrm{mc}}}\left(d_{R_{k} D}\right)^{-\lambda} \widehat{x}_{k}+\eta_{R_{k} D}
$$

in which $R_{\mathrm{mc}}$ and $P_{\mathrm{mc}}$ are the coding rate and power transmitted in the MAC mode, respectively, where the total power transmitted or the DTM power is $P_{\mathrm{DTM}}=P_{b}+P_{\mathrm{mc}}$; $d_{R_{k} D}$ and $\hat{x}$ are the distance and modulated signal transmitted from $R_{k}$ to $D$, respectively.

We analyze the DCCP systems employing coding scheme. The instantaneous signal-to-noise ratio $(\gamma)$ for DTM is given as

$$
\gamma_{\mathrm{DTM}}=R_{\mathrm{DTM}} d_{\mathrm{DTM}} \frac{P_{\mathrm{DTM}}}{N_{o}}\left(d_{\mathrm{SD}}\right)^{-\lambda}
$$

where $R_{\mathrm{DTM}}$ and $d_{\mathrm{DTM}}$ are coding rate and the hamming distance in DTM, respectively. The hamming distance is measure of the error correction capability of the code; that is, if $d$ is high, the capability of correction is high at destination; otherwise it is low. In our analysis, we are interested in coding gain which is equal to the multiplication of the hamming distance by code rate, that is, $R_{\mathrm{DTM}} d_{\mathrm{DTM}}$. However, when the cooperation is employed, the instantaneous $\gamma$ of $\mathrm{BC}$ mode at $D$ is given as

$$
\gamma_{b}=R_{b} d_{b} \frac{P_{b}}{N_{o}}\left(d_{S D}\right)^{-\lambda}
$$

in which $R_{b}$ and $d_{b}$ are coding rate and the hamming distance in BC mode, respectively. In the sequel, the instantaneous $\gamma$ of MAC mode at $D$ is given as

$$
\gamma_{\mathrm{mc}}=\sum_{k=1}^{L} R_{\mathrm{mc}} d_{\mathrm{mc}} \frac{P_{\mathrm{mc}}}{N_{o}}\left(d_{R_{k} D}\right)^{\lambda},
$$

where $R_{\mathrm{mc}}$ and $d_{\mathrm{mc}}$ are coding rate and the hamming distance between received and transmitted signal of the MAC mode, respectively, where $d_{\mathrm{DTM}}=d_{b}+d_{\mathrm{mc}}$ and $R_{\mathrm{DTM}}=$ $\left(R_{b}^{-1}+R_{\mathrm{mc}}^{-1}\right)^{-1}$. Therefore, the total received $\gamma$ at $D$ is given as

$$
\gamma_{\mathrm{CTM}}=R_{b} d_{b} \frac{P_{b}}{N_{o}}\left(d_{S D}\right)^{-\lambda}+\sum_{k=1}^{L} R_{\mathrm{mc}} d_{\mathrm{mc}} \frac{P_{\mathrm{mc}}}{N_{o}}\left(d_{R_{k} D}\right)^{-\lambda} .
$$

2.2. Relays Location Selection. To prevent relays that are two hops away from the destination to participate in the cooperation, we must select the RR, so that only nodes within the RR can participate in the cooperation. In fact, relays positioned two hops away from destination can increase the delay. The proposed protocol is based on CSMA/CA with RTS/CTS packets; in this protocol, when the source has frame to transmit to the destination, if the medium is ideal for the DIFS (DCF interframe space) time, the source transmits a packet Request-To-Send (RTS) to the destination. If the destination is not busy and receives the RTS correctly, it waits for the short interframe space (SIFS) time and transmits back the Clear-To-Send packets (CTS) to the source.

Therefore the RR is selected as follows: after $D$ received the RTS packet, nodes within the source range set timer equal to 2SIFS; during the 2SIFS time, the destination transmits CTS packet. The nodes within the $S$ range that hear CTS can participate in cooperation; otherwise, nodes that do not receive a CTS packet do not participate in the cooperation. As shown in Figure 2, only nodes within the RR can receive RTS/CTS packets, identifying them as one hop away from the destination. It is clear that relays selection procedures are done before the source transmission that we call a proactive selection protocol.

2.3. Backoff Time Evaluation of the Best Relays. In this subsection, we investigate the best relay selection protocol 


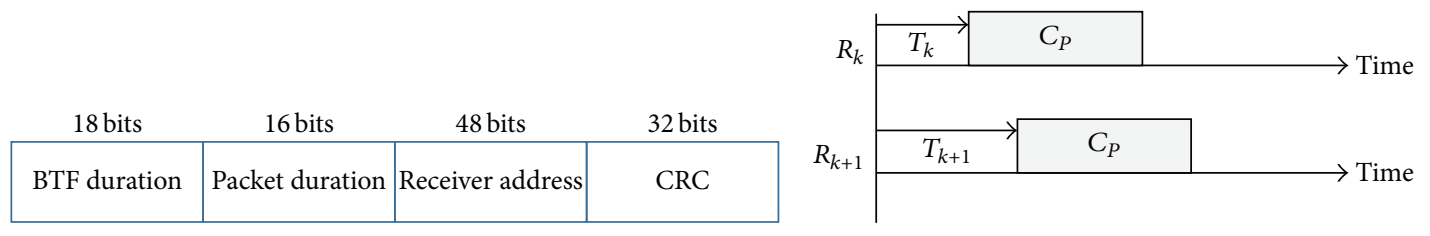

(a)

(b)

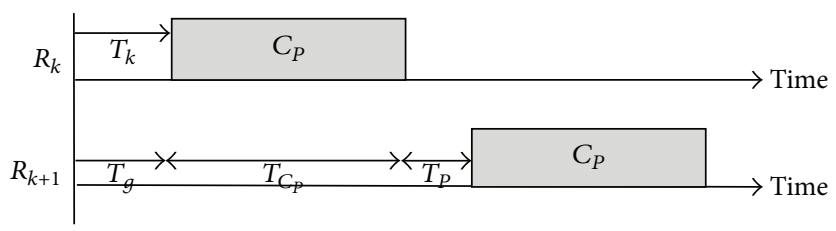

(c)

Figure 3: (a) Control packet $\left(C_{P}\right)$ structure of the proposed protocol. (b) Collision occurred. (c) Collision avoided.

based on BTF to ensure that the best relays access the channel first. Therefore, the BTF is derived as follows: after successful handshaking, in which RTS/CTS packets are received correctly, the source broadcasts the FF with coding rate $R_{b}$ to all nodes within the relayed region. At the relays only correctly received FFs are reencoded with coding rate $R_{\mathrm{mc}}$. After encoding frames with $R_{\mathrm{mc}}$, contention is initiated. In order to ensure that only the relays with the best channel quality toward to the destination access the channel first, we proposed BTF that decreases according to distance and received power quality from the relays to destination. In fact, the reasons behind the BTF were (1) to prevent the relays from using the RTS/CTS packets to access the channel, where the RTS/CTS packets cause extra delay and throughput reduction and (2) to make best relays access the channel first and fast. The BTF mathematically can be expressed as

$$
T_{k}=\left(\left\lfloor\operatorname{RCW}\left(\frac{d_{k, D}}{d_{\mathrm{Thr}}}+\left(1-\frac{S_{\mathrm{Rec}, R_{k}}}{S_{\mathrm{Thr}}}\right)^{-1}\right)\right\rfloor+\mathrm{SIFS}\right),
$$

where $T_{k}$ is the BTF calculated in each relay within RR; $k=1,2, \ldots, L$, where $L$ is the number of relays, RCW is the relay contention windows, $d_{k, D}$ is the instantaneous normalized distance between relay $k$ and the destination, $d_{\mathrm{Thr}}$ is a normalized threshold distance between $R_{k}$ and $D, S_{\text {Thre }}$ is the threshold of the received signal power, and $S_{\text {rec, } R_{k}}=$ $\left(d_{k, D}\right)^{-\lambda}$ is the received signal power at $k$ relays from the destination which is represented by the large scale fading (attenuation). The relays can estimate the received power and distance to destination through the received CTS packet [12].

The backoff time decreases according to the distance and attenuation toward $D$; for example, if RCW $=10 \times(\mathrm{SIFS}=$ $10 \mu \mathrm{s}), d_{\mathrm{Thr}}=1, d_{1, D}=0.25, d_{2, D}=0.9, S_{\mathrm{Thr}}=1$, and $S_{\mathrm{Rec}, 1}=0.0625$ and $S_{\mathrm{Rec}, 2}=0.81$ with $\lambda=3$, we calculate $T_{1}=116 \mu \mathrm{s}$ and $T_{2}=469 \mu \mathrm{s}$. Hence, the relay node with $T_{1}$ has less backoff time to access the channel compared to $T_{2}$. In fact, the BTF depends on the three variables: the distances from $R_{k}$ to $D$ indicated as $d_{R_{k} D}$, received signal power at relays indicated as $S_{\text {rec, } R_{k}}$, and relay contention windows indicated as RCW. $d_{R_{k} D}$ and $S_{\text {rec, } R_{k}}$ depend on the location of the relays from the destination and on the path loss exponents $\lambda$, respectively; however, $\mathrm{RCW}$ is modifiable. The $\mathrm{BTF}$ is directly proportional to the RCW size; there is a question that needs to be answered, what is the suitable RCW size? First of all, let us explain the differences between small and large RCW as follows.

(1) If we set RCW to a small value, that is, $100 \mu \mathrm{s}$, and if two relays have slight differences in distance to the destination, that is, $d_{1, D}=0.2$ and $d_{2, D}=0.3$, then we calculate $T_{1}=130 \mu \mathrm{s}$ and $T_{2}=142 \mu \mathrm{s}$; furthermore, $T_{\max }=\mathrm{RCW} \times 4.6+(\mathrm{SIFS}=10)=470 \mu \mathrm{s}$ and $T_{\text {min }}=\mathrm{RCW} \times 1.2+10=130 \mu \mathrm{s}$, where $T_{\max }$ occurs at $d_{\max , D}=0.9$; however, $T_{\min }$ occurs at $d_{k, D}=0.2$.

(2) If we set RCW to a much larger value, that is, $300 \mu \mathrm{s}$, with the same distances used in point $1, T_{1}=370 \mu \mathrm{s}$ and $T_{2}=406 \mu \mathrm{s}$, then $T_{\max }=\mathrm{RCW} \times 4.6=1390 \mu \mathrm{s}$ and $\mathrm{RCW} \times 1.2=370 \mu \mathrm{s}$.

Let us define the time gap parameter as $T_{g}=T_{k}-T_{(k+1)}$, which is the difference between two BTFs. And it is clear that if the $T_{g}$ is very small meaning that RCW is also very small, the collision may occur if two relays have approximately same distances toward the destination (not equal) due to propagation and control packet time; and if the $T_{g}$ is large since RCW is large, the collision may not occur even when two relays have approximately the same distances toward destination. $T_{g}$ is directly proportional to RCW size; hence, the suitable $T_{g}$ size can draw the suitable RCW size. Therefore, $T_{g}$ is given as

$$
T_{g}=T_{k}-T_{k+1} \quad \text { for } T_{k}-T_{k+1}>T_{\text {Pro }}+T_{C_{P}},
$$

where $T_{k}$ is the first expired BTF, $T_{k+1}$ is the subsequent expired BTF, $T_{\text {Pro }}$ is propagation delay, and $T_{C_{P}}$ is the time due to the control packet transmission; it is explained in the next section, and its value equals $114 \mu \mathrm{s}$. Therefore, we must set RCW to maintain the time gap given (8); in other words, if two relays with a slight difference in distances from the destination and we use a small RCW, relays will have a slight difference in their BTF; in such case collision may occur because of the propagation delay and control packet time, see Figures 3(b) and 3(c). However, if RCW is set to a large value, 
the BTF is large, and the selection may take place on the far relays which make BTF larger; in such case unnecessary delay happens and consequently throughput is reduced. Therefore, choosing RCW size is required to the trade-off between collision occurrence and unnecessary delay.

2.4. Relays Contention and Recalculating BTF of $R_{k+1}$. When more than two relays participate in the cooperation, two relays may have the same backoff time, and a collision may occur. Collisions cannot be prevented in the DWN, but we can ensure that collisions occur between control packet $\left(C_{P}\right)$ rather than data packets $\left(D_{P}\right)$ because retransmission of $D_{P}$ requires more time than $C_{P}$, particularly when the control packet is designed with few number of bits. The difference between the time required for retransmission of data and the control packet is given as

$$
\begin{aligned}
& T_{\text {Retransmission }} \\
& =\left\{\begin{array}{l}
\text { Retrans. of collided data } \\
\approx \text { DIFS }+\frac{D_{P} \text { Size }}{R}+\text { SIFS } \\
\text { Retrans. of collided Control Packet } \\
\approx \frac{C_{P} \text { Size }}{R}+\text { SIFS. }
\end{array}\right.
\end{aligned}
$$

We can describe relays contention as follows: after successful handshaking, the source broadcasts the FF with half redundant bits $N_{b}$ to the destination and relays. Only nodes within the RR and that decode the received FF correctly participate in the cooperation and reencode the SF with half redundant bits $N_{\mathrm{mc}}$. Before the relays start retransmitting to the destination, they calculate their BTF given in (7) according to the available channel parameters from $R_{k}$ to $D$; and the best relay broadcasts the $C_{P}$. During BTF calculation two cases may arise: (1) no collision occurs between relays and $C_{P}$ packets, which refer to the fact that the BTF of each relay is different, that is, $T_{1}<T_{2}<T_{3}<T_{4}<\cdots<T_{k}$ or (2) two relays have same BTF, however, if collisions occurred, which means two BTFs expired together (two relays have the same channel parameters toward destination). The best relay knows whether the collision occurred or not when it transmits $C_{P}$ to the destination and waits for SIFS: if the destination does not reply with 1 bit digit, it means a collision has occurred; otherwise, the collision did not occur. In fact, the collided relays are discarded from the cooperation.

Now let us describe the recalculation of BTF for $R_{k+1}$ as follows: Free from the occurrence of the collision $R_{k+1}$ recalculates their BTF, by decoding the received frame and reading the BTF duration field (see Figure $3(\mathrm{a})$ ). Then, the relays reduce their backoff time by subtracting $T_{k}$ from $T_{k+1}$. However, we must maintain the gap between the two relays since they may have approximately equal distance to the destination that was presented previously, that is, $T_{g}=T_{P}+$ $T_{C_{P}}$. Finally, the updated BTF at $R_{k+1}$ is given as

$$
T_{k+1_{\text {update }}}=\left(T_{k+1}-T_{k}=T_{g}\right)+\left(T_{P}+T_{C_{P}}\right) .
$$

\section{Cooperative Medium Access Delay}

The delay is the time required for a frame to reach to the destination after its transmission from the source, while medium access delay is the time required for the successful transmission and reception after the contention. Finally, cooperative medium access delay is the successful transmission and reception of the transmitted frames from both the source and relays; the queuing delay is not included because our interest is the network delay. In this paper, we consider two situations of delay calculation: successful transmission among a source-relays-destination or collision among all control packets (because we don't have collided data).

\subsection{With and Without Collision Free Cases of the Proposed} Protocol. At the beginning we consider collision-free case. If the source has data to transmit, then it senses the channel, if the channel is free, it starts backoff DIFS, then transmits the RTS to the destination. The destination waits for SIFS then transmits back the CTS packet; after RTS/CTS packets are correctly received, the source broadcasts the FF with half redundant bits. Hence, the total time required to access and broadcast the FF in CTM by the source is given as

$$
T_{b}=\mathrm{DIFS}+T_{\mathrm{RTS}}+T_{\mathrm{CTS}}+2 \mathrm{SIFS}+T_{d_{S}},
$$

where $T_{d_{S}}, T_{\mathrm{RTS}}$, and $T_{\mathrm{CTS}}$ are the time required to transmit the FF from the source to the destination, time required to transmit RTS packet from the source to the destination, and time required to transmit CTS packet from the destination to the source, respectively. After receiving the FF at relays, relays start calculating their BTF according to (7); then, the relay whose BTF expired first (winner relay denoted by $R_{1}$ ) transmits the $C_{P}$; then the destination waits for SIFS, and it broadcasts back 1 bit digit as an acknowledgment of the received $C_{P}$ from the winner relay correctly. $R_{1}$ and $R_{k+1}$ received 1 bit digit. $R_{1}$ transmits SF with half redundant bits to the destination. The time required for a transmission of the $\mathrm{SF}$ in the MAC mode is given as

$$
T_{\mathrm{mc}, 1}=T_{1}+T_{C_{P}}+\operatorname{SIFS}+T_{d_{\mathrm{mc}, R_{1}}},
$$

where $T_{\mathrm{mc}, 1}$ is the total time required for $R_{1}$ to access the channel and transmit SF; $T_{1}$ is the first expired BTF given by (7); $T_{C_{P}}$ is the time required for $C_{P}$ to be transmitted from the relay to the destination; and $T_{d_{\mathrm{mc}, R_{1}}}$ is the time required to transmit SF from $R_{1}$ to the destination, and the other relays in queue have BTF greater than $T_{1}$ (i.e., $T_{1}<T_{2}<T_{3}<T_{4}<$ $\cdots<T_{k}$ ). For the other relays, we do not need $C_{P}$ packet; relays only need to calculate their BTF as well as to the $T_{g}$. Therefore, the required time for the other relays in queue is given as

$$
\begin{aligned}
T_{\mathrm{mc}, k+1}= & (L-1) \operatorname{SIFS}+\sum_{k=1}^{L}\left(T_{k+1}-T_{1}\right) \\
& +\sum_{k=1}^{L} T_{d_{\mathrm{m} c, R_{k+1}}}+T_{C_{P}}+T_{P}+T_{\mathrm{ACK}},
\end{aligned}
$$


where the second term $\left(T_{1}-T_{k+1}\right)$ is BTF of the next relay; we write it in such form to reduce the delay that will occur when the next relay has a BTF much larger than the first BTF; $T_{d_{\mathrm{mc}, R_{k+1}}}$ is time required to transmit SF from $R_{k+1}$ to the destination, and $T_{\mathrm{ACK}}$ is time required for the acknowledgment packet of the whole CTM. Therefore, the total time required for the transmission of the data from the source to the destination over DCCP without collision is found by adding (11), (12), and (13) together, and it is given as

$$
T_{\text {tot,nc }}=T_{b}+T_{\mathrm{mc}, 1}+T_{\mathrm{mc}, k+1} .
$$

If the collision takes place, then in the BC mode it occurs between RTS/CTS packets; therefore, the time required for transmission of the FF to the destination is given as

$$
T_{b, c}=\mathrm{DIFS}+2 T_{\mathrm{RTS}}+2 T_{\mathrm{CTS}}+4 \mathrm{SIFS}+T_{d_{S}} .
$$

We assumed maximum two best relays may have collision (two BTF expired together); the collision takes place between two $C_{P}$ packets; therefore, the time required for transmission of the SF in the MAC mode is given as

$$
T_{\mathrm{mc}, 1, c}=2 T_{1}+2 T_{C_{P}}+2 \text { SIFS }+T d_{\mathrm{mc}, R_{1}} .
$$

Then, the total time required for the transmission of the data from the source to the destination over DCCP under collision assumption is given as

$$
T_{\mathrm{tot}, c}=T_{b, c}+T_{\mathrm{mc}, 1, c}+T_{\mathrm{mc}, k+1} .
$$

The indexes in (14) and (17) are no collision (nc) and collision (c), respectively.

\section{Connectivity via Cooperation Scheme}

The definition of connectivity is the measure of the robustness of links between neighbouring nodes in their vicinity; in other words, it measures the ability of nodes to be connected under specific conditions. Connectivity is an important issue in DWN because it may have isolated nodes that could lead to disconnected nodes or clusters. From a $\gamma$ perspective, two nodes are connected (linked together) if the $\gamma$ is greater than the threshold value; from the graph theory perspective, connectivity measures the possibility of isolated nodes (node outside their groups) or isolated clusters (isolated groups). In this paper, we consider linking probability.

4.1. Linking Probability. Linking probability is the probability of links between two nodes under specific conditions. The linking probability has been defined in detail in $[18,19]$, where the previous work considered a shadowing channel model; in this paper, the channel model is large scale fading (i.e., distance and path loss exponent). If the signal-to-noise ratio of the DTM decreases or begins to approach the threshold value, the linking probability decreases or becomes zero. For a given $\gamma_{\mathrm{DTM}}$ and $\gamma_{\text {Thre }}$ in $\mathrm{dB}$, communication between two nodes is possible when $\gamma_{\mathrm{DTM}} \geq \gamma_{\text {Thre }}$. In this work, we assume that $P\left(\gamma_{\text {DTM }} \geq \gamma_{\text {Thre }}\right)=1$. However, the definition of the linking probability in the CTM is indicated as the probability that $\gamma_{\mathrm{CTM}}$ of the cooperation is greater than or equal to $\gamma_{\mathrm{DTM}}$. Therefore, the linking probability of the CTM is given as

$$
\begin{aligned}
& P_{t}\left(\gamma_{\mathrm{CTM}} \geq \gamma_{\mathrm{DTM}}\right) \\
& \quad=\frac{1}{\sqrt{2 \pi} \sigma} \int_{0}^{\infty} \exp \left[-\frac{1}{2 \sigma^{2}}\left(t-\frac{\gamma_{\mathrm{CTM}}}{\gamma_{\mathrm{DTM}}}\right)^{2}\right] d t, \\
& P_{t}\left(\gamma_{\mathrm{CTM}} \geq \gamma_{\mathrm{DTM}}\right) \\
& \quad=\frac{1}{\sqrt{2 \pi} \sigma} \int_{0}^{\infty} \exp \left[-\frac{1}{2 \sigma^{2}}\left(t-\frac{\gamma_{\mathrm{CTM}}}{\gamma_{\mathrm{DTM}}}\right)^{2}\right] d t .
\end{aligned}
$$

This yields

$$
P_{t}\left(\gamma_{\mathrm{CTM}} \geq \gamma_{\mathrm{DTM}}\right)=0.5\left[1-\operatorname{erf}\left(\frac{\left(\gamma_{\mathrm{CTM}}-\gamma_{\mathrm{DTM}}\right)}{\sqrt{2}}\right)\right] .
$$

Then, we substitute (6) into (19), and then the linking probability of CTM is given as

$$
\begin{aligned}
& P_{t}\left(\gamma_{\mathrm{CTM}} \geq \gamma_{\mathrm{DTM}}\right) \\
&=0.5[1-\operatorname{erf}(0.7071 \\
& \cdot(\left(R_{b} d_{b} \frac{P_{b}}{N_{o}}\left(d_{\mathrm{SD}}\right)^{-\lambda}\right) \\
&\left.\left.\left.\cdot\left(\prod_{k=1}^{L} R_{\mathrm{mc}} d_{\mathrm{mc}} \frac{P_{\mathrm{mc}}}{N_{o}}\left(d_{R_{k} D}\right)^{-\lambda}\right)\right)-\gamma_{\mathrm{DTM}}\right)\right] .
\end{aligned}
$$

4.2. Number of Neighbor Nodes. The number of neighbor nodes is the number of nodes within the coverage area of a node and is called the node degree $(\rho)$. If the node does not have nodes within its coverage area, the node is isolated from the network. In this paper, we are interested in calculating the node degree of random nodes distribution over service area which is given as [18]

$$
\rho_{\mathrm{DTM}}=\omega \int_{0}^{2 \pi} P\left(\gamma_{\mathrm{DTM}} \geq \gamma_{\mathrm{Thre}}\right) d \theta=2 \pi \omega P\left(\gamma_{\mathrm{DTM}} \geq \gamma_{\mathrm{Thre}}\right) \text {, }
$$

where $\omega$ is the node density within a whole area and $\omega=$ $(n / A)$, where $n$ is the number of nodes within the service area and $A$ is the service area size (in meters). Then, the node degree due to the CTM is given as

$$
\begin{aligned}
\rho_{\mathrm{CTM}} & =2 \pi \omega\left(P\left(\gamma_{\mathrm{DTM}} \geq \gamma_{\mathrm{Thre}}\right)+P\left(\gamma_{\mathrm{CTM}} \geq \gamma_{\mathrm{DTM}}\right)\right) \\
& =2 \pi \omega\left(1+P\left(\gamma_{\mathrm{CTM}} \geq \gamma_{\mathrm{DTM}}\right)\right),
\end{aligned}
$$

where $\rho_{\mathrm{DTM}}$ and $\rho_{\mathrm{CTM}}$ are node degrees of DTM and CTM, respectively. The cooperation increases the coverage area of the source (see Figure 4). This is due to increased detection capability of the destination which comes from MRC combining of the received signals, and this results in the increased 


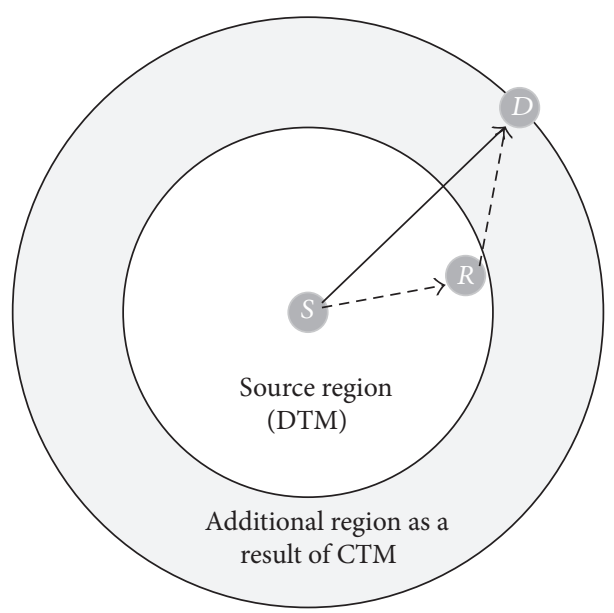

FIGURE 4: The additional region as a result of CTM is shown (extra coverage area gained for the source).

node degree of the source. Now consider the question: Why does the increment in the node degree result in the decrement in throughput? The answer comes from the MAC protocol contention based opinion, where the coverage area shares single time slot and the nodes within the coverage area are assumed to be circularly accessing the channel; therefore, the time slot (throughput) is divided by number of the nodes within the coverage area; hence it increases the node degree leading to throughput reduction. It is also clear that, if $\gamma_{\mathrm{CTM}}=$ $\gamma_{\text {DTM }}$, then $P\left(\gamma_{\text {CTM }}>\gamma_{\text {DTM }}\right)=0$.

\section{Throughput Analysis}

For convenience and to facilitate comprehension, we considered a throughput analysis of the DWN based on an analytical model given in [20]. The throughput $(H)$ is given as

$$
H=\frac{T_{\mathrm{DTM}} W_{\mathrm{CTM}}}{\rho_{\mathrm{CTM}} T_{t, \mathrm{CTM}} E(h)},
$$

where $\rho_{\mathrm{CTM}}$ is the node degree of the CTM; $T_{\mathrm{DTM}}$ is the time required to transmit a frame to the destination over DTM which is given in (11); $T_{t, \mathrm{CTM}}=T_{o}+T_{\mathrm{CTM}}$ is the total time required to transmit frame over CTM, where $T_{\mathrm{CTM}}$ and $T_{o}$ are the time required for transmission over CTM and overhead time, respectively. In addition $T_{\text {CTM }}$ indicate to $T_{\text {tot, }}$ or to $T_{\text {tot,nc }}, W_{\mathrm{CTM}}$ is the upper bound on reliable data transmission (Shannon capacity), and $E(h)$ is the expected number of hops in this work $E(h)=1$. Note that the variables with index CTM are affected by the cooperation of the DCCP, where Shannon capacity of DCCP is given as [21]

$$
\begin{aligned}
W_{\mathrm{CTM}}= & \left(2 B_{\mathrm{DTM}}\right) \\
& \cdot \log _{2}\left(R_{b} d_{b} \frac{P_{b}}{N_{o}}\left(d_{S D}\right)^{-\lambda}+\sum_{k=1}^{L} R_{\mathrm{mc}} d_{\mathrm{mc}} \frac{P_{\mathrm{mc}}}{N_{o}}\left(d_{R_{k} D}\right)^{-\lambda}\right),
\end{aligned}
$$

where $B_{\mathrm{DTM}}$ is the bandwidth of DTM; generally, the coding scheme reduces the bandwidth efficiency by coding rate ratio
$R_{\mathrm{DTM}}=\left(N_{\mathrm{DTM}} / k_{\mathrm{C}}\right)$, in which $N_{\mathrm{DTM}}$ is the number of bits out of the encoder, and $k_{C}$ is the number of input bits to the encoder, where $N>k_{C}$, and $R_{\mathrm{DTM}}$ is the coding rate; for example, if the coding rate is $1 / 4$, then the bandwidth efficiency is reduced by $1 / 4$; therefore, the bandwidth efficiency of CTM is double compared to DTM because the DCCP divides the redundant bits by 2 on the source and relays which results in higher coding rate, $R_{\mathrm{CTM}}=1 / 2$. The bandwidth efficiency of DCCP is given as $\left(N / 2 k_{C}\right) B_{\mathrm{CTM}}=\left(N / k_{C}\right) B_{\mathrm{DTM}}$, and, as a result, $B_{\mathrm{CTM}}=2 B_{\mathrm{DTM}}$. Finally, we can rewrite (23) as

$$
\begin{gathered}
H=\left(T _ { \mathrm { DTM } } ( 2 B _ { \mathrm { DTM } } ) \operatorname { l o g } _ { 2 } \left(R_{b} d_{b} \frac{P_{b}}{N_{o}}\left(d_{\mathrm{SD}}\right)^{-\lambda}\right.\right. \\
\left.\left.+\sum_{k=1}^{L} R_{\mathrm{mc}} d_{\mathrm{mc}} \frac{P_{\mathrm{mc}}}{N_{o}}\left(d_{R_{k} D}\right)^{-\lambda}\right)\right) \\
\cdot\left(T_{t, \mathrm{CTM}} E(h)\left(\mathrm{DIFS}+T_{\mathrm{RTS}}+T_{\mathrm{CTS}}+2 \mathrm{SIFS}+T_{d_{S}}\right)^{-1}\right. \\
\left.\cdot\left(2 \pi \omega\left(1+P\left(\gamma_{\mathrm{CTM}} \geq \gamma_{\mathrm{DTM}}\right)\right)\right)\right)^{-1} .
\end{gathered}
$$

It is clear that the throughput of the DTM may or may not improve because the relays are always repeating what was transmitted by the source and increasing node degree of the source; accordingly we can draw theorem of throughput relation to delay and connectivity.

Theorem 1. For the proposed relays selection protocol with $L \geq$ 1 and the DCCP employed for random nodes distribution over service area, the achievable throughput is given as

$$
H=O\left(\frac{\gamma(L)}{T(L) \rho_{C T M}(L)}\right) .
$$

The theorem expresses the throughput for CTM, that is, it is proved that the throughput of the cooperation is inversely proportional to the delay and node degree, where 


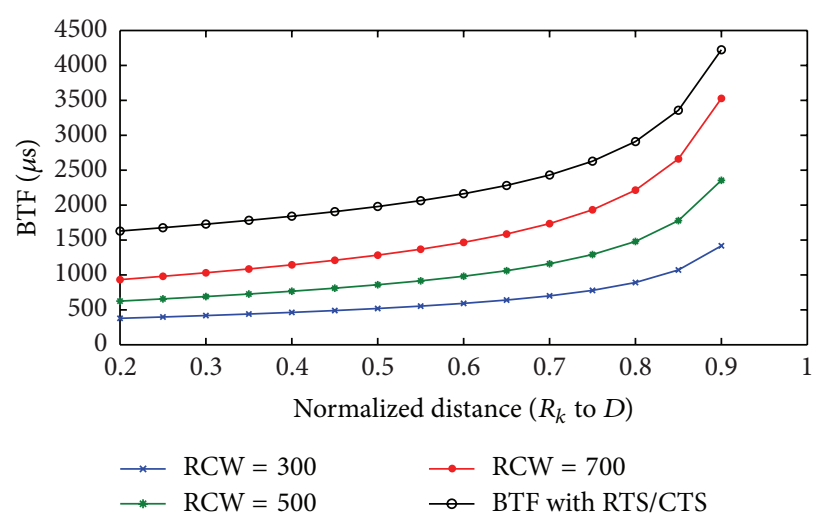

FIGURE 5: Comparison of the BTF and distance from the relays to destination for $L=1$ relay, $\mathrm{RCW}=300,500$, and $750 \mu$ s with traditional CSMA/CA with RTS/CTS packets.

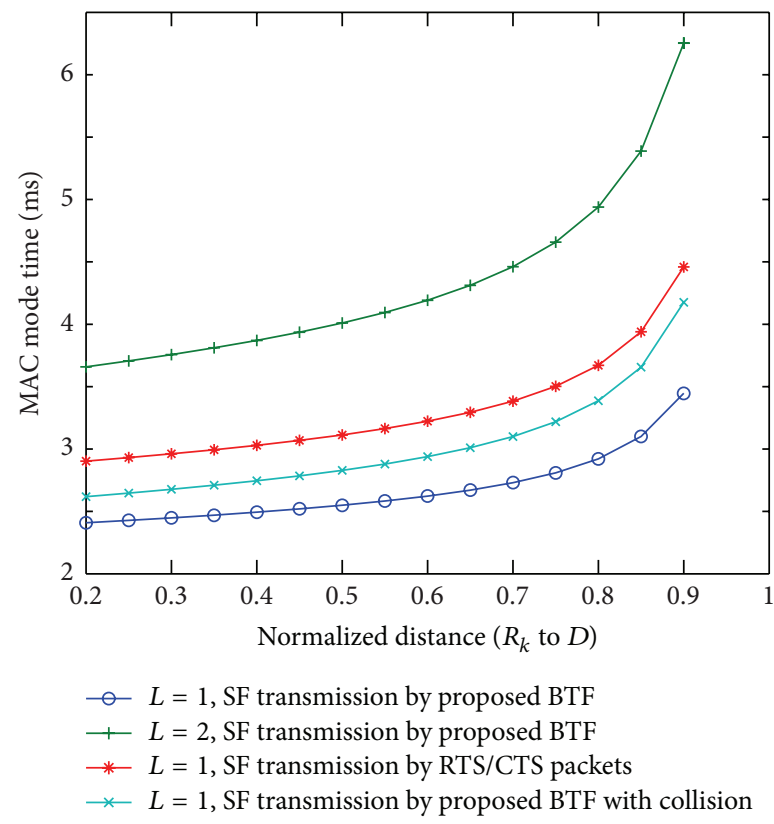

(a)

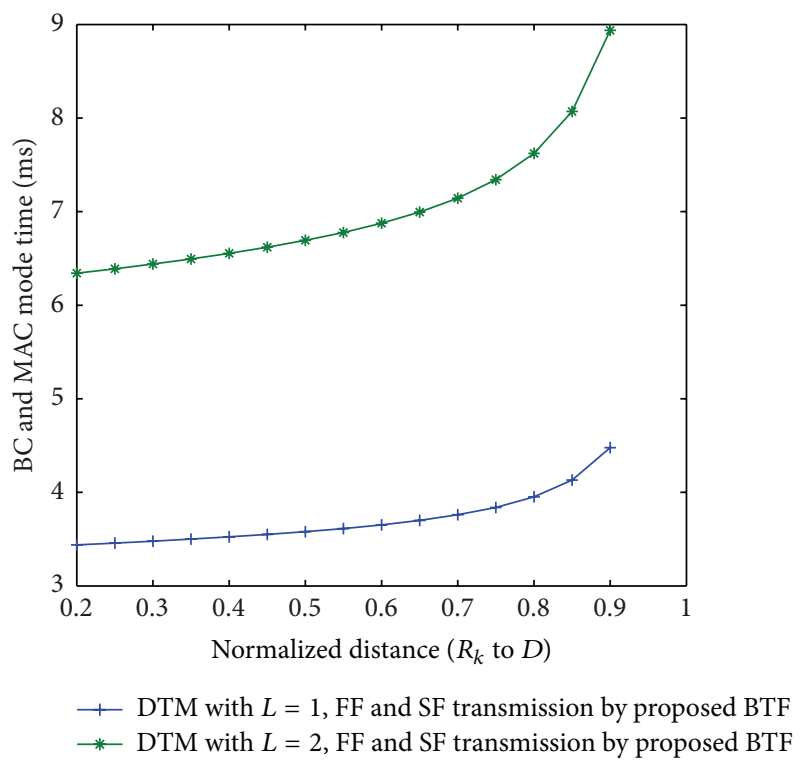

(b)

FIgURE 6: (a) Comparison of required time to transmit SF using proposed protocol and transmission over traditional CSMA/CS with RTS/CTS packets. (b) Comparison of required time to transmit FF and SF, for $L=1,2$ relays, $\mathrm{RCW}=300 \mu \mathrm{s}$, and $\lambda=3$.

$\gamma(L), T(L)$, and $\rho_{\mathrm{CTM}}(L)$ are the signal-to-noise ratio, total time to transmit data from the source to destination over DTM, and node degree, respectively, where $O(\cdot)$ is the big $O$ notation.

\section{Performance and Results}

In this section, we evaluate the performance of the proposed CMAC protocol via an analytical model. The evaluation is divided into three parts: the first part considers the proposed CMAC protocol from a delay perspective; the second part considers connectivity when the DCCP is employed; and finally the third part considers throughput performance as a function of increasing delay and connectivity.
Figure 5 illustrates the BTF variation with distance from the relays to the destination and different RCW size. In fact, as distance from the relays to destination increases, the BTF increases as well, that is lead to deferral of the relays far away from the destination and accelerate the nearest relay to the destination to access the channel. Furthermore, we can see the BTF is less delayed compared to the conventional CSMA/CA with RTS/CTS packets. Therefore, if $d_{k, D}=0.5$, BTF using RTS/CTS packets is $2000 \mu \mathrm{s}$, and RCWs are 300 , 500 , and $750 \mu \mathrm{s}$; accordingly, the BTF of different RCWs is 500,800 , and $1125 \mu$ s, respectively; hence the achieved delay reduction by our proposed protocol compared to BTF using RTS/CTS packets is $300 \%, 150 \%$, and $77.7 \%$. It is clear that the delay reduction is less for larger RCW and high for smaller RCW. 


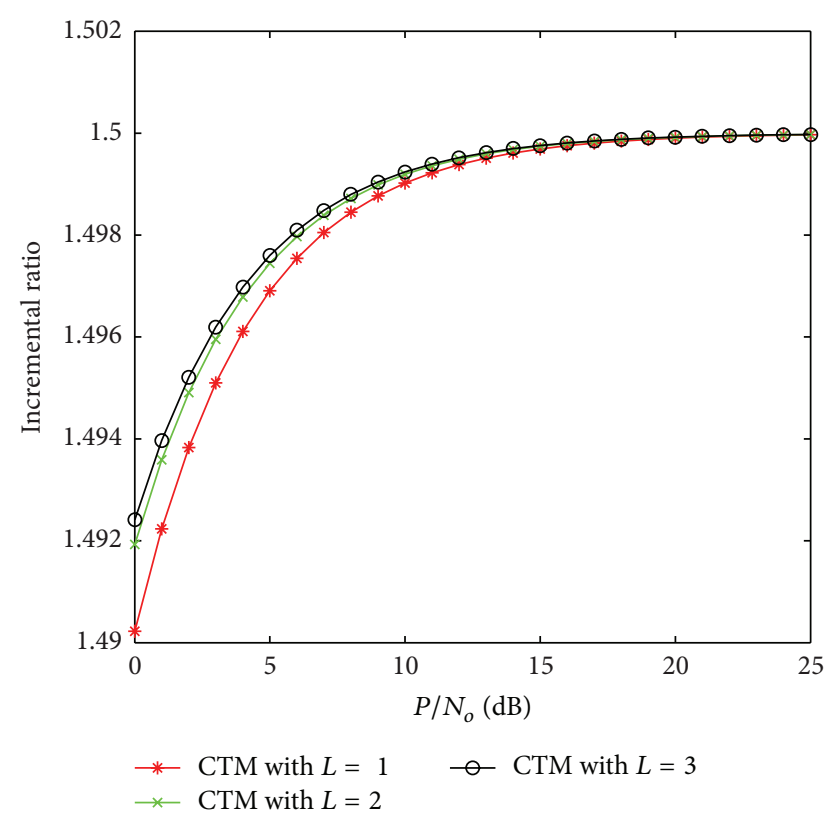

Figure 7: Comparison of the incremental ratio with $P / N_{o}$ for $L=$ $1,2,3$ relays, $\lambda=3, R_{\mathrm{DTM}}=1 / 4$, and $d_{\mathrm{DTM}}=13$.

Figure 6(a) illustrates the SF transmission from the relays to destination using the proposed BTF; in other words, MAC mode transmission time is depicted. It is clear that the transmission of the SF over proposed BTF with and without collision for $L=1$ has less delay compared to the conventional CSMA/CA with RTS/CTS packets. Furthermore, if the two relays participate in the cooperation, then we have too much delay compared to single relay participating in cooperation. In fact, at $d_{k, D}=0.5$, the times required to transmit SF using our proposed protocol are $2.5 \mathrm{~ms}$ and $2.7 \mathrm{~ms}$ for $L=1$ and $L=1$ with collision, respectively, and the time required to transmit SF using RTS/CTS packets is $3 \mathrm{~ms}$; as a result, the achieved delay reduction using our proposed protocol is $16.66 \%$ and $11 \%$. We conclude that the time required to transmit SF using our proposed protocol compared to conventional RTS/CTS packets is less even when the collision occurred.

Figure 6(b) illustrate the total time required for transmission of the FF and SF over BTF using $L=1$ and $L=2$, where the delay increases if the number of relays increases.

Figure 7 illustrate the incremental ratio comparison to power-to-noise ratio $\left(P / N_{o}\right)$. The incremental ratio is the ratio between node degrees of CTM and the node degree of the DTM. As shown, based on the results, the connectivity increased using different number of relays; consequently, the incremental ratio increases as well. Increasing number of relays can increase the connectivity since signal-to-noise ratio increased at destination which can improve the detection capability and connectivity.

Figure 8 illustrate a comparison of the throughput of the proposed relay selection protocol, traditional CSMA/CA with RTS/CTS packets, and number of relays. The important issues are apparent in Figure 8.

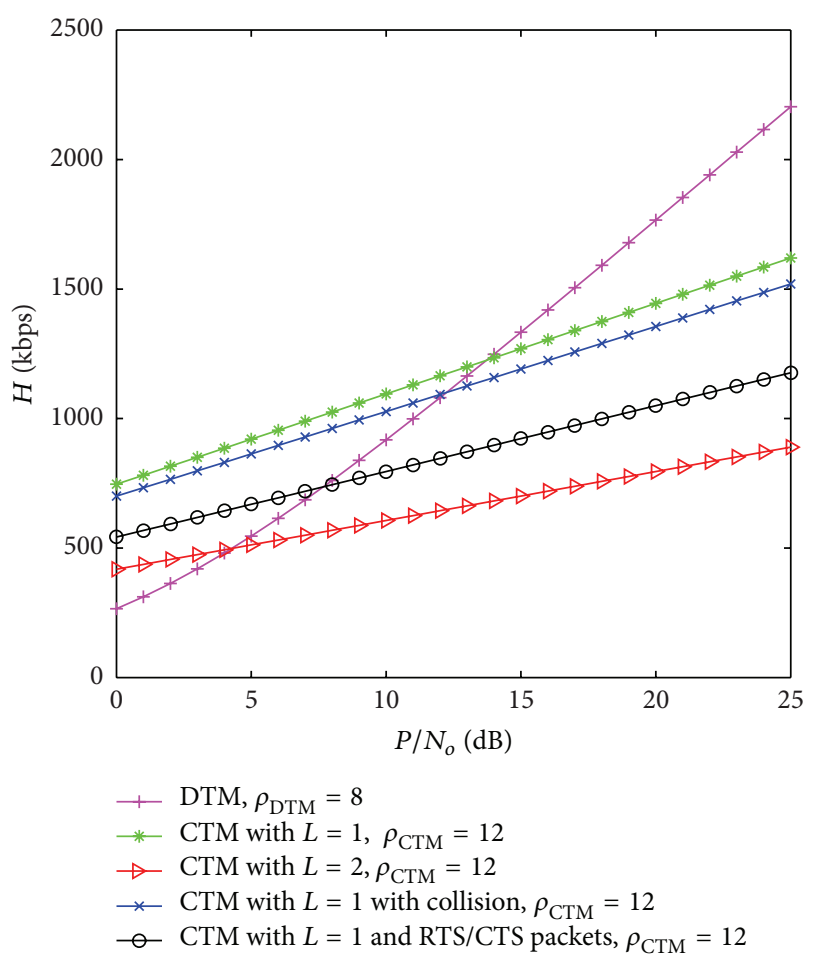

FIGURE 8: Comparison of the throughput of the DTM and CTM for the $L=1,2$ relays, $\rho_{\text {DTM }}=8, \rho_{\text {CTM }}=12, \mathrm{RCW}=300 \mu \mathrm{s}, d_{k, D}=0.5$ and $\lambda=3$.

(1) If the number of the relays increases, the throughput reduces.

(2) The proposed relay selection protocol can achieve better throughput compared to traditional CSMA/CA with RTS/CTS packets.

(3) Increasing number of relay can reduce the throughput compared to single relay.

(4) The throughput of the DTM is better at high $P / N_{o}$ compared to CTM.

In fact, at $P / N_{o}=15 \mathrm{~dB}$, the throughput of DTM is larger compared to our proposed protocol because the delay and node degree increased. On the other hand, the throughput of our proposed protocol is larger for $P / N_{o}$ less than $15 \mathrm{~dB}$ compared to DTM. In addition, the throughput of CTM reduced by ratio $1 /\left(\rho_{\mathrm{CTM}}=12\right)=0.08$, while the throughput of DTM reduced by ratio $1 /\left(\rho_{\mathrm{DTM}}=8\right)=0.125$. The achieved throughput of our proposed protocol is $78 \%$, for $L=1, P / N_{o}=15 \mathrm{~dB}$ with collision-free case compared to conventional RTS/CTS packets. The evaluation parameters and setting are summarized in Table 1.

\section{Conclusion}

In this paper, we have proposed a cooperative MAC protocol for decentralized DWN in which the proposed schemes consider the best single or multiple relays selection, delay, and collision avoidance. We also investigated a BTF; when 
TABLE 1: Evaluation parameters and settings.

\begin{tabular}{lc}
\hline SIFS, DIFS, $T_{\mathrm{CTS}}, T_{\mathrm{ACK}}, T_{\mathrm{RTS}}$ & $10,50,304,304,352(\mu \mathrm{s})$ \\
RCW & $300,500,750(\mu \mathrm{s})$ \\
$R$, transmission rate & $1 \mathrm{Mbps}$ \\
$T_{o}$ (overhead time) for IEEEb [13], $T_{\text {Pro }}$ & $364,5 \mu \mathrm{s}$ \\
(propagation time) & $2000 \mathrm{bits}$ \\
$D_{P}$ size & $114 \mu \mathrm{s}$ \\
$T_{C_{P}}$ & $1 \mathrm{MHz}$ \\
$B_{\mathrm{DTM}}$, bandwidth & 0 to $25 \mathrm{~dB}$ \\
$\left(P / N_{o}\right)$ & $1,0.2$ to 0.9 \\
$d_{S D}, d_{R_{k}, D}$ & 3 \\
$\lambda$, path loss exponent & 1,3 \\
$L_{\text {min }}, L_{\text {max }}$ & $1 / 4,13$ \\
$R_{\mathrm{DTM}}, d_{\mathrm{DTM}}[14]$ & $1 / 2,7,1 / 2,6$ \\
$R_{b}, d_{b}, R_{\mathrm{mc}}, d_{\mathrm{mc}}$ & 21 \\
$n$, number of nodes & $10000 \mathrm{~m}^{2}$ \\
$A$, service area &
\end{tabular}

the channel parameters between the relay and destination are bad, a longer time will be associated with the relay and vice versa. We have demonstrated that connectivity in the cooperation is improved which results in an increased node degree. Finally, we have shown that employing cooperation leads to higher delays and decreased the throughput of a DWN. In future work, we will reduce the delay by using a different frequency channel for source and relays rather than the single channel which is used by the source and relays.

\section{Conflict of Interests}

The authors declare that there is no conflict of interests regarding the publication of this paper.

\section{References}

[1] P. Liu, Z. Tao, S. Narayanan, T. Korakis, and S. S. Panwar, "CoopMAC: a cooperative MAC for wireless LANs," IEEE Journal on Selected Areas in Communications, vol. 25, no. 2, pp. 340-354, 2007.

[2] R. Youssef and A. Graell i Amat, "Distributed serially concatenated codes for multi-source cooperative relay networks," IEEE Transactions on Wireless Communications, vol. 10, no. 1, pp. 253263, 2011.

[3] W. Zhang, Y. Li, N. G. Xia, P. C. Ching, and K. B. Letaief, "Distributed space-frequency coding for cooperative diversity in broadband wireless ad hoc networks," IEEE Transactions on Wireless Communications, vol. 7, no. 3, pp. 995-1002, 2008.

[4] Y. Li, B. Vucetic, T. F. Wong, and M. Dohler, "Distributed turbo coding with soft information relaying in multihop relay networks," IEEE Journal on Selected Areas in Communications, vol. 24, no. 11, pp. 2040-2050, 2006.

[5] Y. Jing and H. Jafarkhani, "Single and multiple relay selection schemes and their achievable diversity orders," IEEE Transactions on Wireless Communications, vol. 8, no. 3, pp. 1414-1423, 2009.
[6] K. Vardhe, D. Reynolds, and B. Woerner, "Joint power allocation and relay selection for multiuser cooperative communication," IEEE Transactions on Wireless Communications, vol. 9, no. 4, pp. 1255-1260, 2010.

[7] A. S. Ibrahim, A. K. Sadek, W. Su, and K. J. R. Liu, "Cooperative communications with relay-selection: when to cooperate and whom to cooperate with?" IEEE Transactions on Wireless Communications, vol. 7, no. 7, pp. 2814-2827, 2008.

[8] N. Tadayon, H. Wang, D. Kasilingam, and L. Xing, "Analytical modeling of medium-access delay for cooperative wireless networks over rayleigh fading channels," IEEE Transactions on Vehicular Technology, vol. 62, no. 1, pp. 349-359, 2013.

[9] X. Wang and J. Li, "Network coding aware cooperative MAC protocol for wireless Ad Hoc networks," IEEE Transactions on Parallel and Distributed Systems, vol. 25, no. 1, pp. 167-179, 2014.

[10] A. El Gamal, J. Mammen, B. Prabhakar, and D. Shah, "Optimal throughput-delay scaling in wireless networks, part II: constant-size packets," IEEE Transactions on Information Theory, vol. 52, no. 11, pp. 5111-5116, 2006.

[11] O. Dousse and P. Thiran, "Connectivity vs capacity in dense ad hoc networks," in Proceedings of the 23rd Annual Joint Conference of the IEEE Computer and Communications Societies (INFOCOM '04), vol. 1, May 2004.

[12] A. Bletsas, A. Khisti, D. P. Reed, and A. Lippman, "A simple cooperative diversity method based on network path selection," IEEE Journal on Selected Areas in Communications, vol. 24, no. 3, pp. 659-672, 2006.

[13] Part 11: Wireless LAN Medium Access Control (MAC) and Physical Layer (PHY) Specification, IEEE Std. 802.11-2012, 1999.

[14] P. Frenger, P. Orten, and T. Ottosson, "Convolutional codes with optimum distance spectrum," IEEE Communications Letters, vol. 3, no. 11, pp. 317-319, 1999.

[15] G. Bianchi, "Performance analysis of the IEEE 802.11 distributed coordination function," IEEE Journal on Selected Areas in Communications, vol. 18, no. 3, pp. 535-547, 2000.

[16] M. Elfituri, W. Hamouda, and A. Ghrayeb, "A convolutionalbased distributed coded cooperation scheme for relay channels," IEEE Transactions on Vehicular Technology, vol. 58, no. 2, pp. 655-669, 2009.

[17] J. G. Proakis, Digital Communication, McGraw-Hill, New York, NY, USA, 1995.

[18] C. Bettstetter and C. Hartmann, "Connectivity of wireless multihop networks in a shadow fading environment," Wireless Networks, vol. 11, no. 5, pp. 571-579, 2005.

[19] R. Hekmat and P. van Mieghem, "Connectivity in wireless adhoc networks with a log-normal radio model," Mobile Networks and Applications, vol. 11, no. 3, pp. 351-360, 2006.

[20] R. Hekmat and P. van Mieghem, "Interference in wireless multi-hop ad-hoc networks and its effect on network capacity," Wireless Networks Journal, vol. 10, no. 4, pp. 389-399, 2004.

[21] G. Ferrari and O. K. Tonguz, "MAC protocols and transport capacity in ad hoc wireless networks: Aloha versus PR-CSMA," in Proceedings of the IEEE Military Communications Conference (MILCOM), vol. 2, Boston, Mass, USA, October 2003. 


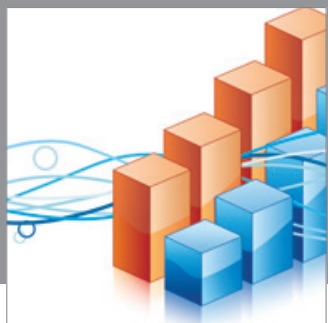

Advances in

Operations Research

mansans

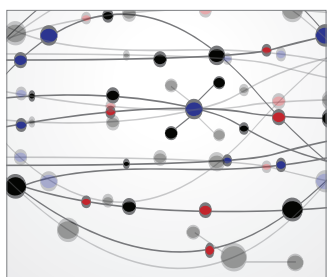

The Scientific World Journal
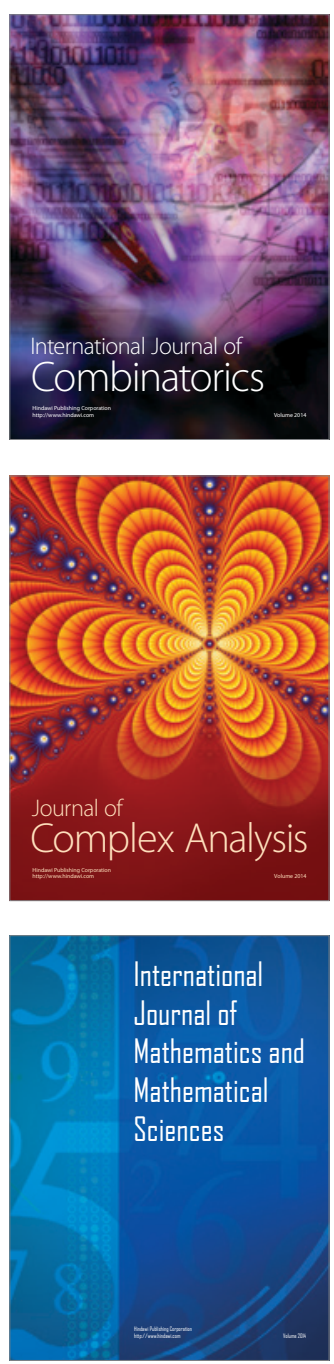
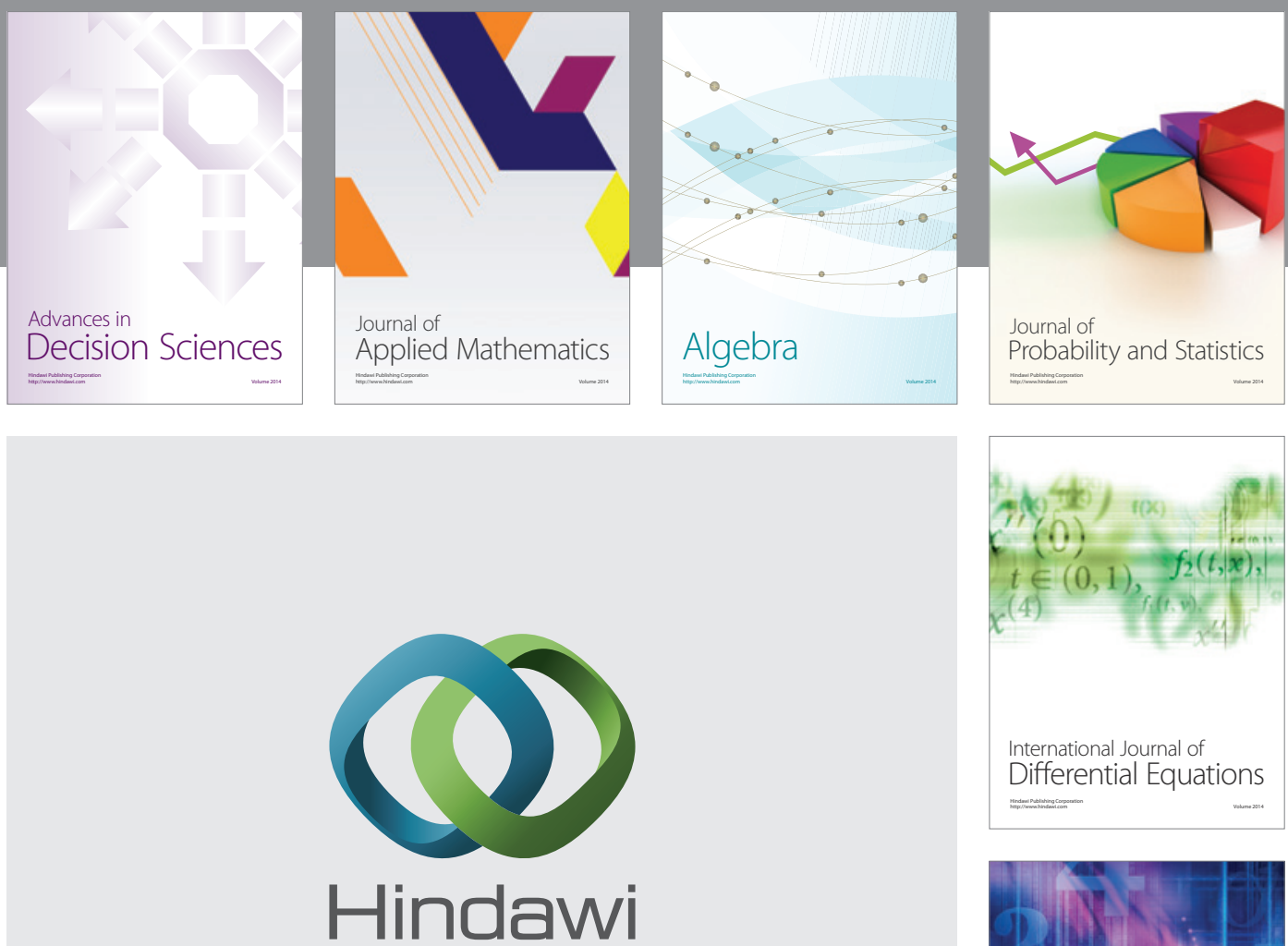

Submit your manuscripts at http://www.hindawi.com
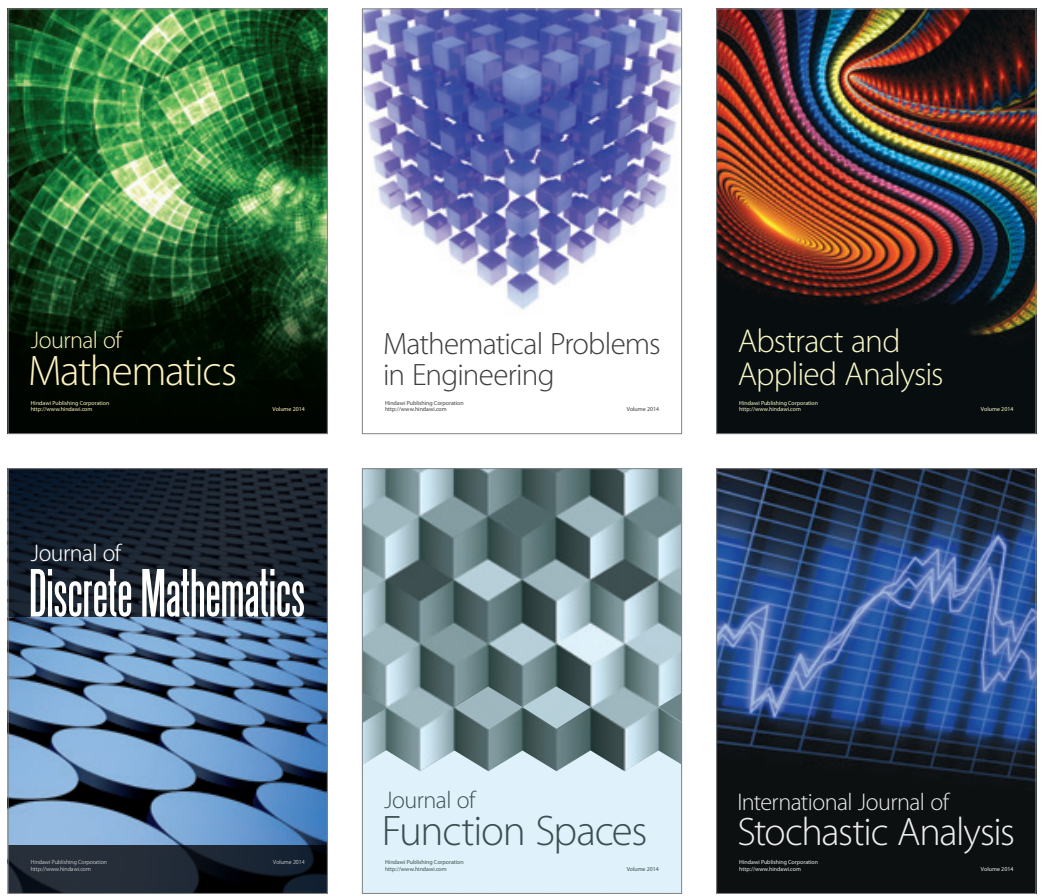

Journal of

Function Spaces

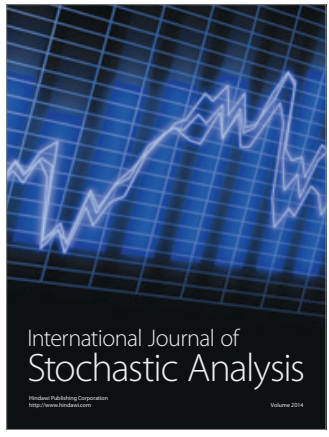

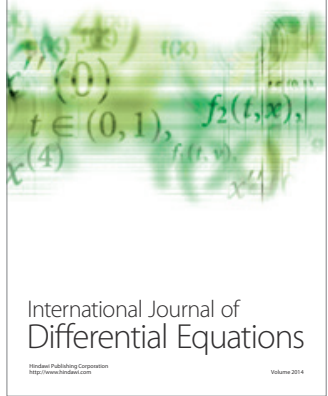
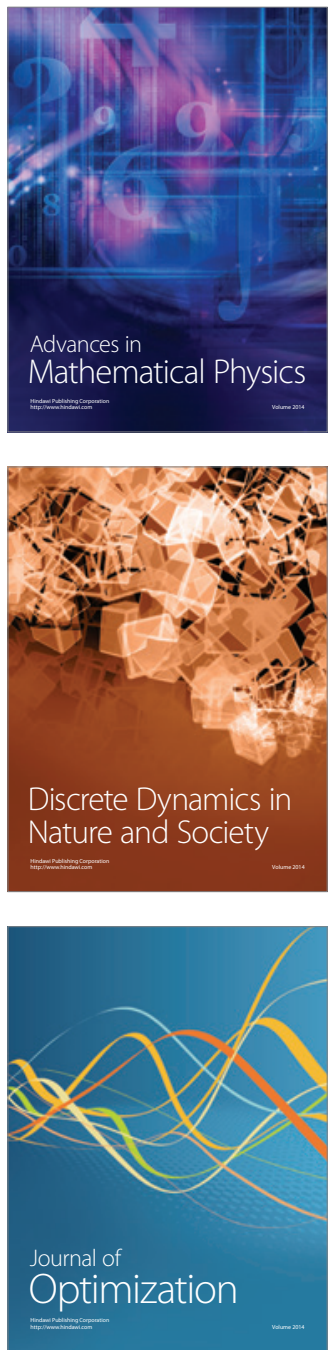